\title{
Peak-to-Average Power Ratio Reduction for Wavelet Packet Modulation Schemes via Basis Function Design
}

\author{
Ngon Thanh Le ${ }^{1}$, Siva D. Muruganathan ${ }^{2}$ and Abu B. Sesay ${ }^{1}$ \\ ${ }^{1}$ Department of Electrical $\&$ Computer Engineering, University of Calgary, \\ ${ }^{2}$ Department of Electrical $\mathcal{E}$ Computer Engineering, University of Alberta
}

Canada

\section{Introduction}

Orthogonal frequency division multiplexing (OFDM) is a well known and widely employed high-data rate transmission technology. By dividing the wideband channel into multiple narrowband sub-channels, OFDM mitigates the detrimental effects of the multi-path fading channel. A main drawback of OFDM is that the transmitted signal possesses a high peak-toaverage power ratio (PAPR). Since the OFDM modulated signal is the superposition of many constituent narrowband signals, it is possible for these constituent narrowband signals to align in phase and produce high peak values. This results in high PAPRs where the peak power of the OFDM modulated signal is very large compared to the average power. For applications employing highly power-efficient power amplifiers, the input signal with a high PAPR causes the power amplifier to be operated in the non-linear region. The non-linear characteristic of the high power amplifier (HPA) will introduce in-band distortions which in turn will degrade system performance. Additionally, a HPA operated in the non-linear region will also produce out-of-band power emissions which will generate spectral regrowth and produce unwanted interference to the adjacent channel users. To alleviate the high PAPR problem in OFDM systems, several PAPR reduction schemes have been proposed in the literature (see Han \& Lee, 2005; Jiang \& Wu, 2008, and references therein).

Wavelet packet modulation (WPM) is an alternative multi-carrier technology that has received recent research attention (see Lakshmana \& Nikookar, 2006 and references therein). WPM systems have been shown to have better immunity to impulse and narrowband noises than OFDM (Lindsey, 1995). Furthermore, WPM systems attain better bandwidth efficiency than OFDM (Sandberg \& Tzannes, 1995), and they do not require cyclic prefix extension unlike OFDM (Lakshmana \& Nikookar, 2006). To benefit from these advantages, WPM has been recently applied in the various areas including multi-carrier multi-code code division multiple access (CDMA) (Akho-Zhieh \& Ugweje, 2008), cognitive radio systems (Lakshmanan, Budiarjo, \& Nikookar, 2007), and multiple-input multiple-output systems (Lakshmanan, Budiarjo, \& Nikookar, 2008), to name a few. However, similar to OFDM, WPM also suffers from the PAPR problem.

The objectives of this chapter are twofold. Firstly, we provide an overview of the PAPR reduction methods already published in the literature for WPM systems. As part of the 
overview, we discuss the merits and limitations associated with the existing PAPR reduction schemes. Secondly, we formulate the design criteria for a set of PAPR minimizing orthogonal basis functions for WPM systems. To evaluate the merits of the PAPR minimizing orthogonal basis functions, performance comparisons are made with the conventional Daubechies basis functions and OFDM. Furthermore, we also provide a qualitative comparison between the PAPR minimizing orthogonal basis functions and the existing PAPR reduction techniques for WPM systems.

The rest of the chapter is organized as follows. First, an overview of WPM based multicarrier systems is provided in Section 2. An overview of some PAPR reduction methods already proposed in the literature for WPM systems is then presented in Section 3. Next, in Section 4 , we present the design criteria of the PAPR minimizing orthogonal basis functions. This is followed by simulation results and discussions in Section 5. Finally, the chapter is concluded in Section 6.

The following notations are used throughout the chapter: $E\{\theta\}$ denotes the statistical average of random variable $\theta ; \operatorname{Pr}\left\{\theta>\theta_{0}\right\}$ represents the probability of the event $\theta>\theta_{0}$; $\max _{n}\{\tilde{\theta}(n)\}$ represents the maximum value of $\tilde{\theta}(n)$ over all instances of time index $n$; $\operatorname{sign}\{\bullet\}$ is the signum function; $\delta(p)$ represents the Kronecker delta function; $\lceil\theta\rceil$ denotes the smallest integer greater than or equal to $\theta ; \forall$ represents universal quantification.

Parts of this work is based on "An Efficient PAPR Reduction Method for Wavelet Packet Modulation Schemes", by N. T. Le, S. D. Muruganathan, and A. B. Sesay which appeared in IEEE Vehicular Technology Conference, Spring-2009. The previously published material is reused with permission from IEEE. Copyright [2009] Institute of Electrical and Electronics Engineers.

\section{Overview of WPM based multi-carrier systems}

In general, the WPM based multi-carrier systems can be characterized using sets of orthogonal basis functions called wavelet packets (Lakshmanan \& Nikookar, 2006). These orthogonal basis functions are defined at multiple levels with the $m^{\text {th }}$ level consisting of $2^{m}$ distinct basis functions. Let us denote the orthogonal basis functions corresponding to the $m^{\text {th }}$ level by the set

$$
\left\{\varphi_{m, 0}(t), \quad \varphi_{m, 1}(t), \quad \ldots, \quad \varphi_{m, k}(t) \quad \ldots, \quad \varphi_{m, 2^{m}-2}(t), \quad \varphi_{m, 2^{m}-1}(t)\right\},
$$

where $k\left(k=0,1, \ldots, 2^{m}-1\right)$ denotes the orthogonal basis function index. Given (1), the orthogonal basis functions corresponding to the $(m+1)^{\text {th }}$ level are defined as

$$
\begin{gathered}
\varphi_{m+1,2 k}(t)=\sum_{n} \tilde{h}(n) \varphi_{m, k}(2 t-n), \\
\varphi_{m+1,2 k+1}(t)=\sum_{n} \tilde{g}(n) \varphi_{m, k}(2 t-n),
\end{gathered}
$$

where $\tilde{h}(n)$ and $\tilde{g}(n)$ respectively denote low-pass and high-pass filter impulse responses forming a quadrature mirror filter (QMF) pair at the receiver. Hence, $\tilde{h}(n)$ and $\tilde{g}(n)$ are related through (Lakshmanan \& Nikookar, 2006) 


$$
\tilde{g}(n)=(-1)^{n} \tilde{h}(2 N-1-n),
$$

where $2 \mathrm{~N}$ denotes length of both filters. In addition, the low-pass filter impulse response $\tilde{h}(n)$ is also required to satisfy the following conditions (Daubechies, 1992):

$$
\begin{gathered}
\sum_{n} \tilde{h}(n) \tilde{h}(n+2 p)=\delta(p), \\
\sum_{n} \tilde{h}(n)=\sqrt{2} .
\end{gathered}
$$

Let us next define the time-reversed version of the QMF pair at the transmitter as follows:

$$
\begin{aligned}
& h(n)=\tilde{h}(-n), \\
& g(n)=\tilde{g}(-n),
\end{aligned}
$$

where $h(n)$ and $g(n)$ represent the time-reversed low-pass and high-pass filter impulse responses, respectively.

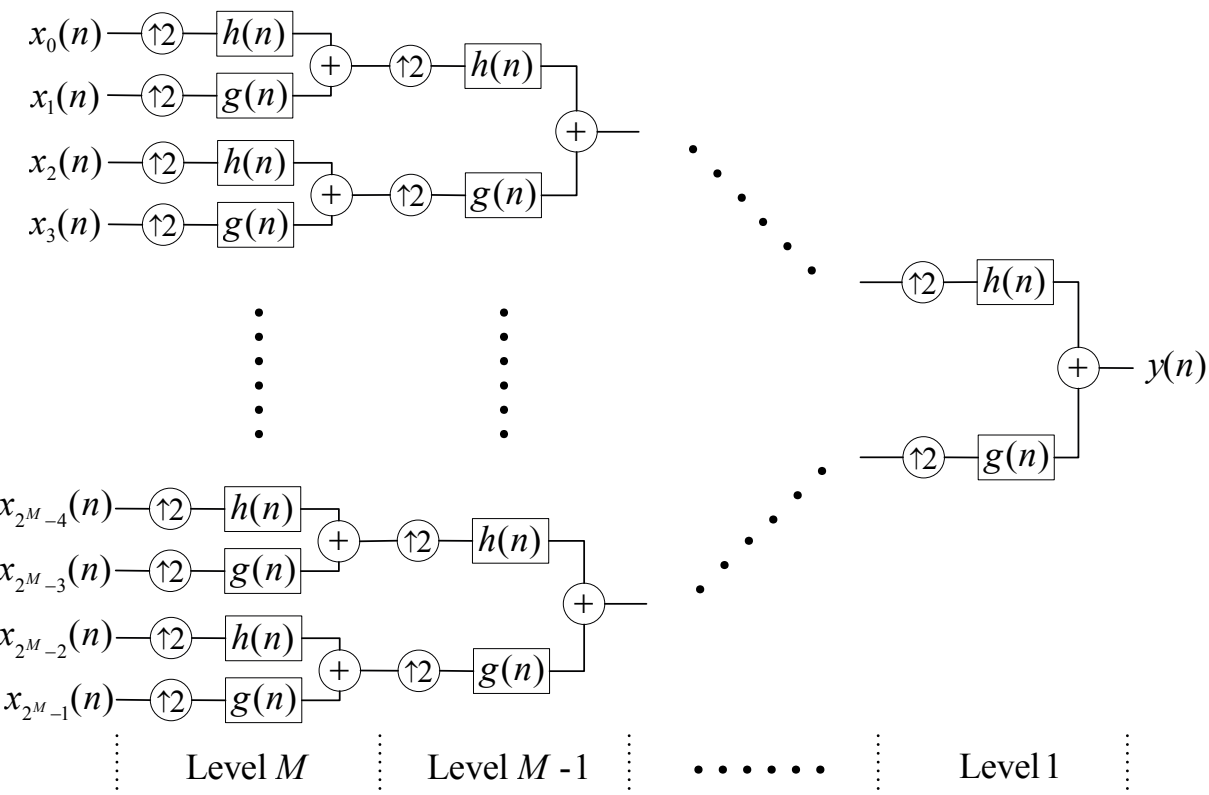

Fig. 1. A $2^{M}$ sub-channel WPM transmitter structure

Now, the transmitter structure for a WPM multi-carrier system with $2^{M}$ sub-channels can be constructed as shown in Figure 1. In Figure 1, the input data symbol streams $x_{0}(n), \quad x_{1}(n), \ldots, x_{2^{M}-2}(n), \quad x_{2^{M}-1}(n)$ are multiplexed onto $2^{M}$ sub-channels via the successive application of time-reversed QMF pairs (i.e., $h(n)$ and $g(n))$. For a system with $2^{M}$ sub-channels, we require $M$ levels of QMF pairs at the transmitter, wherein the $m^{\text {th }}$ 
level consists of $2^{m-1}$ QMF pairs. Generally, the successive application of the $M$ levels of QMF pairs yields the output $y(n)$ of the WPM transmitter. It should be noted that the WPM transmitter structure of Figure 1 is also referred to as the wavelet packet tree (WPT) in the literature. Additionally, the combination of the up-sampler and the filter (i.e., either $h(n)$ or $g(n))$ is sometimes called a node.

Alternatively, the WPM transmitter structure of Figure 1 can also be represented as in Figure 2 (Daly et al., 2002). In Figure 2, the equivalent impulse response corresponding to the $k^{\text {th }}\left(k=0,1, \ldots, 2^{M}-1\right)$ sub-channel is denoted as $w_{k}(n)$. Next, let us represent the Ztransform of the filter impulse response corresponding to the $k^{\text {th }}$ sub-channel and the $m^{\text {th }}$ level (refer to Figure 1) as

$$
T_{k, m}(z) \in\{H(z), G(z)\},
$$

where $H(z)$ and $G(z)$ denote the Z-transforms of $h(n)$ and $g(n)$, respectively. Then, the Ztransform of the equivalent impulse response $w_{k}(n)$ is given by (Daly et al., 2002)

$$
W_{k}(z)=\prod_{m=1}^{M} T_{k, m}\left(z^{2^{m-1}}\right)
$$

\section{PAPR reduction techniques for WPM systems}

Although PAPR reduction techniques have been extensively studied for OFDM systems (see Han \& Lee, 2005, Jiang \& Wu, 2008, and references therein), PAPR reduction methods for WPM systems have only recently gained the attention of the Communications and Signal Processing research communities. In this section, we provide an overview of some PAPR reduction methods already proposed in the literature for WPM systems. In doing so, we discuss the merits and limitations of the existing PAPR reduction schemes for WPM systems. This section also helps set the stage for later sections by emphasizing the motivation for proposing a basis function design based PAPR reduction scheme for WPM systems.

\subsection{Tree pruning based methods}

In the tree pruning approach, adjacent nodes of the wavelet packet tree (WPT) are selectively combined (or split) to form a single node (or two separate nodes) in order to reduce the PAPR. Baro and Ilow recently proposed a tree pruning based PAPR reduction scheme where a sequence of modulated symbols are first passed through $N_{T}$ distinct pruned WPTs to produce $N_{T}$ different output sequences (Baro \& Ilow, 2007a). The $N_{T}$ distinct pruned WPTs are attained by performing a single adjacent node-pair joining at different locations of the unpruned WPT (i.e., a single adjacent node-pair joining is performed at $N_{T}$ different locations of the unpruned WPT). Then, the sequence with the lowest PAPR among the $N_{T}$ different output sequences is chosen for transmission. Additionally, to facilitate recovery of the information symbols, details about the pruned WPT chosen for transmission is sent to the receiver as side information via additional subcarriers. 


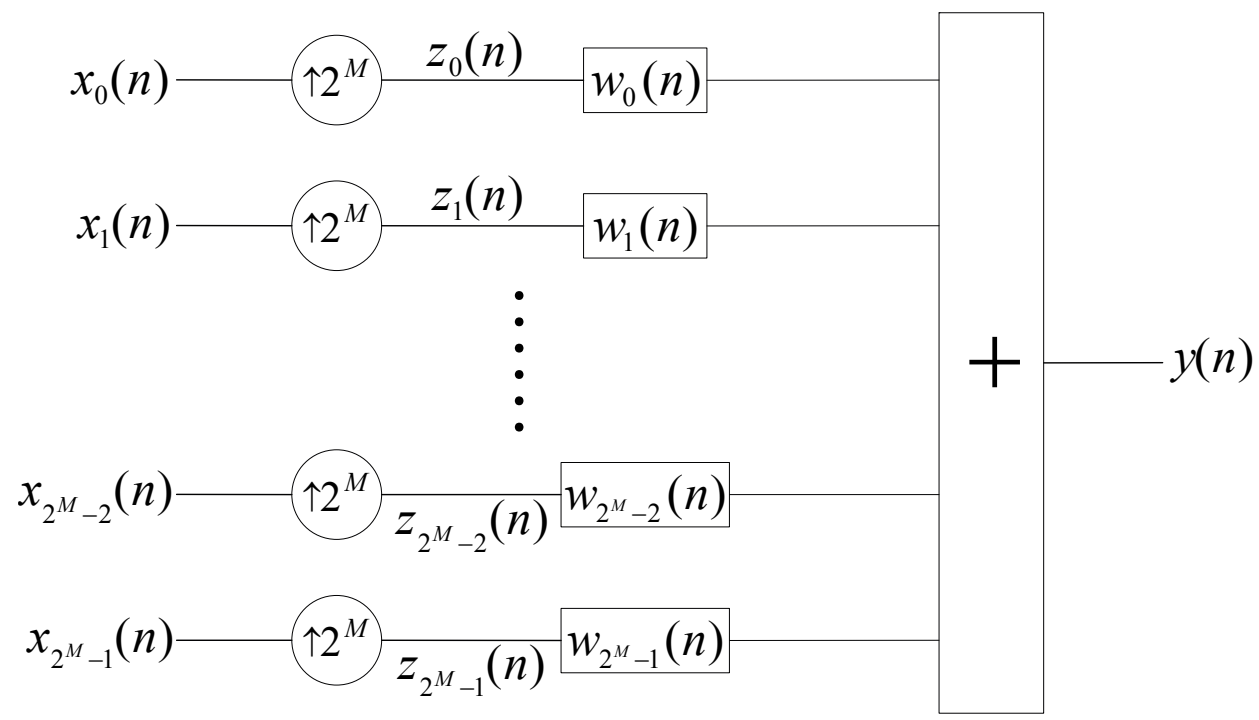

Fig. 2. An equivalent representation of the $2^{M}$ sub-channel WPM transmitter structure

Since the scheme proposed in (Baro \& Ilow, 2007a) requires an exhaustive search for the output sequence with the lowest PAPR, the number $N_{T}$ of pruned WPTs determines the computational complexity associated with it. Furthermore, the number $N_{T}$ also affects the amount of side information required since a minimum of $\left\lceil\log _{2}\left(N_{T}\right)\right\rceil$ bits are necessary in order for the receiver to identify the pruned WPT that was used by the transmitter. To alleviate the complexity and side information overhead issues, the authors in (Baro \& Ilow, 2007a) also propose two low-complexity pruning algorithms. In the first low-complexity algorithm, the authors reduce the number $N_{T}$ of pruned WPTs by choosing only the most frequently chosen $N_{T}^{\prime}$ pruned WPTs (i.e., the ones that generate the output sequence with the lowest PAPR most frequently) obtained through several random simulation trials. This scheme not only reduces the complexity associated with the original tree pruning algorithm but also decreases the side information overhead. The second low-complexity algorithm involves setting a PAPR threshold $P A P R_{t h}$, where the first pruned WPT that produces an output PAPR below $P A P R_{t h}$ is chosen for transmission. Depending on the values of $N_{T}^{\prime}$ and $P A P R_{\text {th }}$ chosen, the two low-complexity versions are shown to slightly reduced PAPR reduction performance (Baro \& Ilow, 2007a).

In (Baro \& Ilow, 2007b), an alternative tree pruning is proposed for PAPR reduction in WPM systems. As opposed to the scheme in (Baro \& Ilow, 2007a) which is based on a single adjacent node-pair joining approach, the scheme proposed in (Baro \& Ilow, 2007b) allows multiple joins on multiple non-leaf nodes. In the later scheme, a set $P^{(D)}$ of allowable nonleaf nodes are first defined, and the joining of multiple nodes is performed iteratively. Since this scheme involves searching for a pruned WPT that yields the least PAPR via multiple iterations (or passes), the authors refer to it as the multi-pass tree pruning method. In reducing the processing time and computational complexity, it is shown in (Baro \& Ilow, 2007b) that the multi-pass tree pruning method provides good PAPR reduction capability 
when the number of iterations (or passes) is set to 5 . Furthermore, a computationally simpler version of the multi-pass tree pruning algorithm based on setting a PAPR threshold $P A P R_{t h}$ is also proposed in (Baro \& Ilow, 2007b). In the simplified version, the iterative search procedure is terminated once a pruned WPT that yields a PAPR value below $P A P R_{t h}$ is found. For the receiver to identify the pruned WPT that was used by the transmitter, the multi-pass pruning scheme requires a minimum of $\left\lceil\log _{2}\left(\operatorname{size}\left\{P^{(D)}\right\} \times N_{i}\right)\right]$ bits as side information. Here, size $\left\{P^{(D)}\right\}$ and $N_{i}$ respectively denote the size of the allowable non-leaf node set and the maximum number of iterations. It should be noted that with increasing size $\left\{P^{(D)}\right\}$, the multi-pass pruning scheme yields improved PAPR reduction capability and requires more side information overhead.

The major advantage of tree pruning based methods is their capability to provide high PAPR reduction. The results presented in (Baro \& Ilow, 2007a) show that the single nodepair joining based tree pruning method can yield up to $3.5 \mathrm{~dB}$ of PAPR reduction when compared to the WPM scheme using the unpruned WPT. Likewise, the multi-pass tree pruning method of (Baro \& Ilow, 2007b) can provide up to $5 \mathrm{~dB}$ PAPR reduction over the unpruned WPM scheme. Furthermore, the tree pruning based PAPR reduction methods are distortionless and do not introduce spectral regrowth. The major drawbacks of the tree pruning based PAPR reduction methods are twofold. Firstly, the tree pruning approach requires side information to be sent to the receiver which reduces the bandwidth efficiency of the system. Moreover, when the side information is received in error, the receiver will not be able to recover the transmitted data sequence. Hence, some form of protection such as the employment of channel encoding may be necessary to reliably receive the side information. However, the employment of channel encoding in the transmission of side information will result in further loss of bandwidth efficiency. The second major disadvantage of tree pruning based PAPR reduction methods is that the pruned WPTs result in sub-channels with different bandwidths. Hence, the application of tree pruning based approach does not guarantee that all sub-channels undergo frequency-flat fading. This will reduce the multipath resilience of the WPM system under broadband communication environments.

\subsection{Clipping and amplitude threshold based methods}

The clipping method is the simplest and one of the widely used PAPR reduction methods in multicarrier communication systems (Jiang \& Wu, 2008). Using the clipping method, any desired amount of PAPR reduction can be achieved by presetting the clipping level at the transmitter. In (Rostamzadeh \& Vakily, 2008), a clipping based PAPR reduction scheme is investigated with application to WPM. To reduce the effect of nonlinear distortion introduced by the clipping process, the authors in (Rostamzadeh \& Vakily, 2008) adopt an iterative maximum likelihood (ML) based approach at the receiver. In this approach, estimates of the transmitted symbols are first attained via the ML detector. These transmitted symbol estimates are then used to compute an estimate of the nonlinear distortion component. Next, the nonlinear component estimate is removed from the received signal, and revised ML estimates of the transmitted symbols are obtained. The revised ML estimates of the transmitted symbols are once again used to attain a revised estimate of the nonlinear distortion component. The processes of revised nonlinear distortion component estimation and revised ML estimation of the transmitted symbols are repeated iteratively until a desired level of performance is attained. Bit error rate (BER) 
results presented in (Rostamzadeh \& Vakily, 2008) show that the iterative ML receiver based clipping approach can nearly mitigate the in-band distortion introduced by the clipping process with 3 iterations in an additive white Gaussian noise (AWGN) channel. However, in a multipath fading channel, it is generally difficult to estimate the nonlinear distortion component at the receiver (Jiang \& Wu, 2008). Thus, the iterative ML receiver based clipping approach proposed in (Rostamzadeh \& Vakily, 2008) may suffer performance degradation due to in-band distortion in a multipath fading environment. Other major disadvantages of the iterative ML receiver based clipping approach include increased outof-band radiation and a higher receiver complexity.

In (Zhang, Yuan, \& Zhao, 2005), an amplitude threshold based method is proposed for PAPR reduction in WPM systems. In this method, the signal samples whose amplitudes are below a threshold $T$ are set to zero, and the samples with amplitudes exceeding $T$ are unaltered. Since setting the low amplitude samples to zero is a nonlinear process, this method also suffers from in-band distortion and introduces out-of-band power emissions. Another major disadvantage with the amplitude threshold based PAPR reduction scheme proposed in (Zhang, Yuan, \& Zhao, 2005) is that it results in an increase in the average power of the modified signal. Although the PAPR is reduced due to an increased average power, this method will result in BER degradation when the transmitted signal is normalized back to its original signal power level (Han \& Lee, 2005). Furthermore, the amplitude threshold based PAPR reduction scheme also requires HPAs with large linear operation regions (Jiang \& Wu, 2008). Lastly, the criterion for choosing the threshold value $T$ is not defined in (Zhang, Yuan, \& Zhao, 2005), and hence, may depend on the characteristics of the HPA.

An alternative amplitude threshold based scheme called adaptive threshold companding transform is proposed in (Rostamzadeh, Vakily, \& Moshfegh, 2008). Generally, the application of nonlinear companding transforms to multicarrier communication systems are very useful since these transforms yield good PAPR reduction capability with low implementation complexity (Jiang \& $\mathrm{Wu}, 2008$ ). In the adaptive threshold companding scheme proposed (Rostamzadeh, Vakily, \& Moshfegh, 2008), signal samples with amplitudes higher than a threshold $T$ are compressed at the transmitter via a nonlinear companding function; the signal samples with amplitudes below $T$ are unaltered. To undo the nonlinear companding transform, received signal samples corresponding to signal samples that underwent compression at the transmitter are nonlinearly expanded at the receiver. The threshold value $T$ is determined adaptively at the transmitter and sent to the receiver as side information. Specifically, $T$ is determined adaptively to be a function of the median and the standard deviation of the signal. By compressing the signal samples with high amplitudes (i.e., amplitudes exceeding $T$ ), the adaptive threshold companding scheme achieves notable PAPR reductions in WPM systems. Results presented in (Rostamzadeh, Vakily, \& Moshfegh, 2008) show that the adaptive threshold companding scheme yields a significantly enhanced symbol error rate performance over the clipping method in an AWGN channel. However, the authors in (Rostamzadeh, Vakily, \& Moshfegh, 2008) do not provide performance results corresponding to the multipath fading environment. The received signal samples to be nonlinearly expanded are identified by comparing the received signal amplitudes to the threshold value $T$ at the receiver. Although this approach works reasonably well in the AWGN channel, it may not be practical in a multipath fading channel due to imperfections associated with fading mitigation techniques such as non-ideal channel estimation, equalization, interference cancellation, etc. Another 
major disadvantage associated with the adaptive threshold companding scheme is that it is very sensitive to channel noise. For instance, the larger the amplitude compression at the transmitter, the higher the BER (or symbol error rate) at the receiver due to noise amplification. Furthermore, the adaptive threshold companding scheme suffers a slight loss in bandwidth efficiency due to side information being transmitted from the transmitter to the receiver. The application of this scheme will also result in additional performance loss if the side information is received in error.

\subsection{Other methods}

In (Gautier et al., 2008), PAPR reduction for WPM based multicarrier systems is studied using different pulse shapes based on the conventional Daubechies wavelet family. Simulation results presented in this work show that the employment of wavelet packets can yield notable PAPR reductions when the number of subchannels is low. However, to improve PAPR, the authors in (Gautier et al., 2008) increase the wavelet index of the conventional Daubechies basis functions which results in an increased modulation complexity.

In (Rostamzadeh \& Vakily, 2008), two types of partial transmit sequences (PTS) methods are applied to reduce PAPR in WPM systems. The first method is a conventional PTS scheme where the input signal block is partitioned into $\Phi$ disjoint sub-blocks. Each of the $\Phi$ disjoint sub-blocks then undergoes inverse discrete wavelet packet transformations to produce $\Phi$ different output signals. Next, the transformed output signals are rotated by different phase factors $b_{\phi}(\phi=1,2, \ldots, \Phi)$. The rotated and transformed output signals are lastly combined to form the transmitted signal. In the conventional PTS scheme, the phase factors $\left\{b_{\phi}\right\}$ are chosen such that the PAPR of the combined signal (i.e., the signal to be transmitted) is minimized. The second PTS method applied to WPM systems in (Rostamzadeh \& Vakily, 2008) is the sub-optimal iterative flipping technique which was originally proposed in (Cimini \& Sollenburger, 2000). In the iterative flipping technique, the phase factors $\left\{b_{\phi}\right\}$ are restricted to the values \pm 1 . The iterative flipping PTS scheme first starts off with phase factor initializations of $b_{\phi}=+1$ for $\forall \phi$ and a calculation of the corresponding PAPR (i.e., the PAPR corresponding to the case $b_{\phi}=+1, \forall \phi$ ). Next, the phase factor $b_{1}$ is flipped to -1 , and the resulting PAPR value is calculated again. If the new PAPR is lower than the original PAPR, the phase factor $b_{1}=-1$ is retained. Otherwise, the phase factor $b_{1}$ is reset to its original value of +1 . This phase factor flipping procedure is then applied to the other phase factors $b_{2}, b_{3}, \ldots, b_{\Phi}$ to progressively reduce the PAPR. Furthermore, if a desired PAPR is attained after applying the phase factor flipping procedure to $b_{\phi}(2 \leq \phi<\Phi)$, the algorithm can be terminated in the middle to reduce the computational complexity associated with the iterative flipping PTS technique.

In general, the PTS based methods are considered important for their distortionless PAPR reduction capability in multi-carrier systems (Jiang \& Wu, 2008; Han \& Lee, 2005). The amount of PAPR reduction achieved by PTS based methods depends on the number $\Phi$ of disjoint sub-blocks and the number $W$ of allowed phase factor values. However, the PAPR reduction achieved by PTS based methods come with an increased computational complexity. When applied to WPM systems, PTS based methods require $\Phi$ inverse discrete wavelet packet transformations at the transmitter. Moreover, the conventional PTS scheme incurs a high computational complexity in the search for the optimal phase factors. Another 
disadvantage associated with the PTS based methods is the loss in bandwidth efficiency due to the need to transmit side information about the phase factors from the transmitter to the receiver. The minimum number of side information bits required for the conventional PTS scheme and the iterative flipping PTS scheme are $\left\lceil\Phi \log _{2}(W)\right\rceil$ and $\Phi$, respectively (Jiang $\& \mathrm{Wu}, 2008$; Han \& Lee, 2005). It should also be noted that the PTS schemes will yield degraded system performance if the side-information bits are received in error at the receiver.

Another method considered for reducing the PAPR of WPM systems in (Rostamzadeh \& Vakily, 2008) is the selective mapping (SLM) approach. In the SLM method, the input sequence is first multiplied by $U$ different phase sequences to generate $U$ alternative sequences. Then, the $U$ alternative sequences are inverse wavelet packet transformed to produce $U$ different output sequences. This is followed by a comparison of the PAPRs corresponding to the $U$ output sequences. The output sequence with the lowest PAPR is lastly selected for transmission. To recover the original input sequence at the receiver, side information about the phase sequence that generated the output sequence with the lowest PAPR must be transmitted to the receiver. The PAPR reduction capability of the SLM method depends on the number $U$ of phase sequences considered and the design of the phase sequences (Han \& Lee, 2005). Similar to the PTS method, the SLM approach is distortionless and does not introduce spectral regrowth. The major disadvantages of the SLM method are its high implementation complexity and the bandwidth efficiency loss it incurs due to the requirement to transmit side information. When applied to WPM systems, the SLM method requires $U$ inverse discrete wavelet packet transformations at the transmitter. Furthermore, a minimum of $\left\lceil\log _{2}(U)\right\rceil$ side-information bits are required to facilitate recovery of the original input sequence at the receiver (Jiang \& Wu, 2008; Han \& Lee, 2005). Similar to the PTS based methods, the SLM approach will also degrade system performance if the side-information bits are erroneously received at the receiver.

\section{Orthogonal basis function design approach for PAPR reduction}

In this section, we present a set of orthogonal basis functions for WPM-based multi-carrier systems that reduce the PAPR without the abovementioned disadvantages of previously proposed techniques. Given the WPM transmitter output signal $y(n)$, the PAPR is defined as

$$
P A P R \triangleq \frac{\max _{n}\left\{|y(n)|^{2}\right\}}{E\left\{|y(n)|^{2}\right\}},
$$

where $\max _{n}\{\bullet\}$ represents the maximum value over all instances of time index $n$. The PAPR reduction method presented here is based on the derivation of an upper bound for the PAPR. With regards to (11), we showed in (Le, Muruganathan, \& Sesay, 2008) that

$$
E\left\{|y(n)|^{2}\right\}=\sigma_{x}^{2}
$$

where $\sigma_{x}^{2}$ is the average power of any one of the input data symbol streams $x_{0}(n), \quad x_{1}(n), \ldots, x_{2^{M}-2}(n), \quad x_{2^{M}-1}(n)$. In Section 4.1, we complete the derivation of the 
PAPR upper bound by deriving an upper bound for the peak power $\max _{n}\left\{|y(n)|^{2}\right\}$. The design criteria for the orthogonal basis functions that minimize the PAPR upper bound are then presented in Section 4.2.

\subsection{Upper bound for PAPR}

Let us first consider the derivation of an upper bound for the peak power $\max _{n}\left\{|y(n)|^{2}\right\}$. Using the notation introduced in Figure 2, the WPM transmitter output signal $y(n)$ can be expressed as

$$
y(n)=\sum_{k=0}^{2^{M}-1} \sum_{p} z_{k}(p) w_{k}(n-p),
$$

where $z_{k}(n)$ is the up-sampled version of $x_{k}(n)$ which is defined as

$$
z_{k}(n)= \begin{cases}x_{k}\left(\frac{n}{2^{M}}\right), & \text { if } \bmod \left(n, 2^{M}\right)=0 \\ 0, & \text { otherwise }\end{cases}
$$

Now, substituting (14) into (13), it can be shown that

$$
y(n)=\sum_{k=0}^{2^{M}-1} \sum_{p} x_{k}(p) w_{k}\left(n-2^{M} p\right) .
$$

We next apply the triangular inequality to (15) and obtain the following upper bound for $|y(n)|$ :

$$
|y(n)| \leq \max _{n, k}\left\{\left|x_{k}(n)\right|\right\}\left[\sum_{k=0}^{2^{M}-1} \sum_{p}\left|w_{k}\left(n-2^{M} p\right)\right|\right] .
$$

In (16), $\max _{n, k}\left\{\left|x_{k}(n)\right|\right\}$ denotes the peak amplitude of the input data symbol stream $x_{k}(n)$ over all sub-channels (i.e., $\forall k$ ) and all instances of time index $n$. Hence, from (16), the peak value of $|y(n)|$ over all instances of $n$ can be upper bounded as

$$
\max _{n}\{|y(n)|\} \leq \max _{n, k}\left\{\left|x_{k}(n)\right|\right\} \times \max _{n}\left\{\sum_{k=0}^{2^{M}-1} \sum_{p}\left|w_{k}\left(n-2^{M} p\right)\right|\right\},
$$

where the notation $\max _{n}\{\bullet\}$ is as defined in (11). It can be shown that in (17), equality holds if and only if the input data symbol streams in (15) (i.e., $x_{k}(p)$ for $\forall k$ ) satisfy the condition

$$
x_{k}(p)=\max _{n, k}\left\{\left|x_{k}(n)\right|\right\} \times \operatorname{sign}\left\{w_{k}\left(n-2^{M} p\right)\right\} \times e^{j \alpha},
$$

where $\alpha$ denotes an arbitrary phase value.

Now, using the result in (17), the upper bound for the peak power $\max _{n}\left\{|y(n)|^{2}\right\}$ is attained as 


$$
\max _{n}\left\{|y(n)|^{2}\right\} \leq \max _{n, k}\left\{\left|x_{k}(n)\right|^{2}\right\} \times\left[\max _{n}\left\{\sum_{k=0}^{2^{M}-1} \sum_{p}\left|w_{k}\left(n-2^{M} p\right)\right|\right\}\right]^{2} .
$$

Lastly, substitution of (12) and (19) into (11) yields the PAPR upper bound as

$$
P A P R \leq P A P R_{U B}=\frac{\max _{n, k}\left\{\left|x_{k}(n)\right|^{2}\right\}}{\sigma_{x}^{2}}\left[\max _{n}\left\{\sum_{k=0}^{2^{M}-1} \sum_{p}\left|w_{k}\left(n-2^{M} p\right)\right|\right\}\right]^{2} .
$$

\subsection{Design criteria for PAPR minimizing orthogonal basis functions}

Considering (20), we first note that the new PAPR upper bound is the product of two factors. The first factor

$$
\frac{\max _{n, k}\left\{\left|x_{k}(n)\right|^{2}\right\}}{\sigma_{x}^{2}}
$$

is only dependent on the input data symbol streams $x_{0}(n), \quad x_{1}(n), \quad \ldots, \quad x_{2^{M}-2}(n), \quad x_{2^{M}-1}(n)$. By virtue of (9)-(10), the second factor

$$
\left[\max _{n}\left\{\sum_{k=0}^{2^{M}-1} \sum_{p}\left|w_{k}\left(n-2^{M} p\right)\right|\right\}\right]^{2}
$$

is solely determined by the reversed QMF pair, $h(n)$ and $g(n)$. Furthermore, since $h(n)$ and $g(n)$ are closely related through (7)-(8) and (4), the abovementioned second factor can be expressed entirely in terms of $h(n)$. Recalling from (2)-(8) that the orthogonal basis functions are characterized by the time-reversed low-pass filter impulse response $h(n)$, we now strive to minimize the PAPR upper bound in (20) by minimizing the cost function

$$
C F_{M}=\max _{n}\left\{\sum_{k=0}^{2^{M}-1} \sum_{p}\left|w_{k}\left(n-2^{M} p\right)\right|\right\}
$$

by appropriately designing $h(n)$.

Firstly, let $H(\omega)$ denote the Fourier transform of the low-pass filter impulse response $h(n)$ with length $2 N$. Given a set of orthogonal basis functions with regularity $L \quad(1 \leq L \leq N)$, the magnitude response corresponding to $h(n)$ can be written as (Burrus, Gopinath, \& Guo, 1998)

$$
|H(\omega)|^{2}=\left[\cos ^{2}(\omega / 2)\right]^{L} P\left(\sin ^{2}(\omega / 2)\right)
$$

where

$$
P\left(\sin ^{2}(\omega / 2)\right)=\sum_{\ell=0}^{L-1}\left(\begin{array}{c}
L-1+\ell \\
\ell
\end{array}\right)\left[\sin ^{2}(\omega / 2)\right]^{\ell}+\left[\sin ^{2}(\omega / 2)\right]^{L} R(\cos (\omega)) .
$$


In (23), $R(\cos (\omega))$ denotes an odd polynomial defined as

$$
R(\cos (\omega))= \begin{cases}0, & \text { if } L=N, \\ \sum_{i=1}^{N-L} a_{i}(\cos (\omega))^{2 i-1}, & \text { if } 1 \leq L<N .\end{cases}
$$

It should be noted that the first case (i.e., $L=N$ ) of (24) corresponds to the case of the conventional Daubechies basis functions. In the second case of (24) where $1 \leq L<N$, the coefficients $\left\{a_{i}\right\}$ are chosen such that

$$
P\left(\sin ^{2}(\omega / 2)\right) \geq 0
$$

for $0 \leq \sin ^{2}(\omega / 2) \leq 1$. Now, substituting (24) into (23) yields

$$
P\left(\sin ^{2}(\omega / 2)\right)=\sum_{\ell=0}^{L-1}\left(\begin{array}{c}
L-1+\ell \\
\ell
\end{array}\right)\left[\sin ^{2}(\omega / 2)\right]^{\ell}+\left[\sin ^{2}(\omega / 2)\right]^{L} \sum_{i=1}^{N-L} a_{i}(\cos (\omega))^{2 i-1} .
$$

Since $0 \leq \sin ^{2}(\omega / 2) \leq 1$, we note that

$$
\left[\sin ^{2}(\omega / 2)\right]^{\ell} \geq\left[\sin ^{2}(\omega / 2)\right]^{L}
$$

for $\ell=0,1, \ldots,(L-1)$. Then, using (27), the first term on the right hand side of (26) can be lower bounded as

$$
\sum_{\ell=0}^{L-1}\left(\begin{array}{c}
L-1+\ell \\
\ell
\end{array}\right)\left[\sin ^{2}(\omega / 2)\right]^{\ell} \geq\left[\sin ^{2}(\omega / 2)\right]^{L} \sum_{\ell=0}^{L-1}\left(\begin{array}{c}
L-1+\ell \\
\ell
\end{array}\right)
$$

Next, noting that $\left|a_{i}(\cos (\omega))^{2 i-1}\right| \leq\left|a_{i}\right|$, we have

$$
a_{i}(\cos (\omega))^{2 i-1} \geq-\left|a_{i}\right|
$$

for $i=1,2, \ldots,(N-L)$. Using (29), the second term on the right hand side of (26) can be lower bounded as

$$
\left[\sin ^{2}(\omega / 2)\right]^{L} \sum_{i=1}^{N-L} a_{i}(\cos (\omega))^{2 i-1} \geq-\left[\sin ^{2}(\omega / 2)\right]^{L} \sum_{i=1}^{N-L}\left|a_{i}\right|
$$

Now, combining (28) and (30) with (26) yields

$$
\begin{aligned}
P\left(\sin ^{2}(\omega / 2)\right) & =\sum_{\ell=0}^{L-1}\left(\begin{array}{c}
L-1+\ell \\
\ell
\end{array}\right)\left[\sin ^{2}(\omega / 2)\right]^{\ell}+\left[\sin ^{2}(\omega / 2)\right]^{L} \sum_{i=1}^{N-L} a_{i}(\cos (\omega))^{2 i-1} \\
& \geq\left[\sin ^{2}(\omega / 2)\right]^{L} \sum_{\ell=0}^{L-1}\left(\begin{array}{c}
L-1+\ell \\
\ell
\end{array}\right)-\left[\sin ^{2}(\omega / 2)\right]^{L} \sum_{i=1}^{N-L}\left|a_{i}\right| .
\end{aligned}
$$


Recalling the constraint $P\left(\sin ^{2}(\omega / 2)\right) \geq 0$ from (25), we can further lower bound (31) as

$$
P\left(\sin ^{2}(\omega / 2)\right) \geq\left[\sin ^{2}(\omega / 2)\right]^{L} \sum_{\ell=0}^{L-1}\left(\begin{array}{c}
L-1+\ell \\
\ell
\end{array}\right)-\left[\sin ^{2}(\omega / 2)\right]^{L} \sum_{i=1}^{N-L}\left|a_{i}\right| \geq 0 .
$$

From the last inequality of (32), we have

$$
\sum_{i=1}^{N-L}\left|a_{i}\right| \leq \sum_{\ell=0}^{L-1}\left(\begin{array}{c}
L-1+k \\
k
\end{array}\right) .
$$

Next, using (33), the range of values for coefficient $a_{1}$ is chosen as $-A_{1} \leq a_{1} \leq A_{1}$, where

$$
A_{1}=\sum_{\ell=0}^{L-1}\left(\begin{array}{c}
L-1+k \\
k
\end{array}\right)
$$

Likewise, the ranges of the remaining coefficients $a_{i}(i=2,3, \ldots, N-L)$ are set as $-A_{i} \leq a_{i} \leq A_{i}$, wherein

$$
A_{i}=\sum_{\ell=0}^{L-1}\left(\begin{array}{c}
L-1+k \\
k
\end{array}\right)-\sum_{k=1}^{i-1}\left|a_{k}\right|
$$

Then, each coefficient $a_{i}$ is searched within its respective range in predefined intervals. For each given set of coefficients $\left\{a_{i}\right\}$, the associated cost function $C F_{M}$ is computed using (4), (7)-(10), and (21). Lastly, the time-reversed low-pass filter impulse response $h(n)$ that minimizes the PAPR upper bound of (20) is determined by choosing the set of coefficients $\left\{a_{i}\right\}$ that minimizes the cost function $C F_{M}$ of (21). It should be noted that the cost function $C F_{M}$ of (21) is independent of the input data symbol streams. Hence, using the presented method the impulse response $h(n)$ can first be designed offline and then be employed even in real-time applications.

\section{Simulation results and discussions}

In this section, we present simulation results to evaluate the performance of the PAPR reduction method of Section 4. Throughout this section, we compare the performance of the PAPR minimizing orthogonal basis functions (which are determined by the time-reversed impulse response $h(n)$ designed in Section 4.2) to the performance of the conventional Daubechies basis functions. Additionally, we also make performance comparisons with multi-carrier systems employing OFDM. Throughout the simulations, the number of subchannels in all three multi-carrier systems is set to 64 (i.e., $M=6$ ), and the channel bandwidth is assumed to be $22 \mathrm{MHz}$. Furthermore, the input data symbol streams $x_{0}(n), \quad x_{1}(n), \ldots, \quad x_{2^{M}-2}(n), \quad x_{2^{M}-1}(n)$ are drawn from a 4-QAM symbol constellation. In the cases of the proposed orthogonal basis functions and conventional Daubechies basis functions, we set $N=6$. Furthermore, for the PAPR minimizing orthogonal basis functions, the regularity $L$ is chosen to be 3 (recall that for the conventional Daubechies basis functions $L=N=6$ ). 
The first performance metric we consider is the complementary cumulative distribution function (CCDF) which is defined as

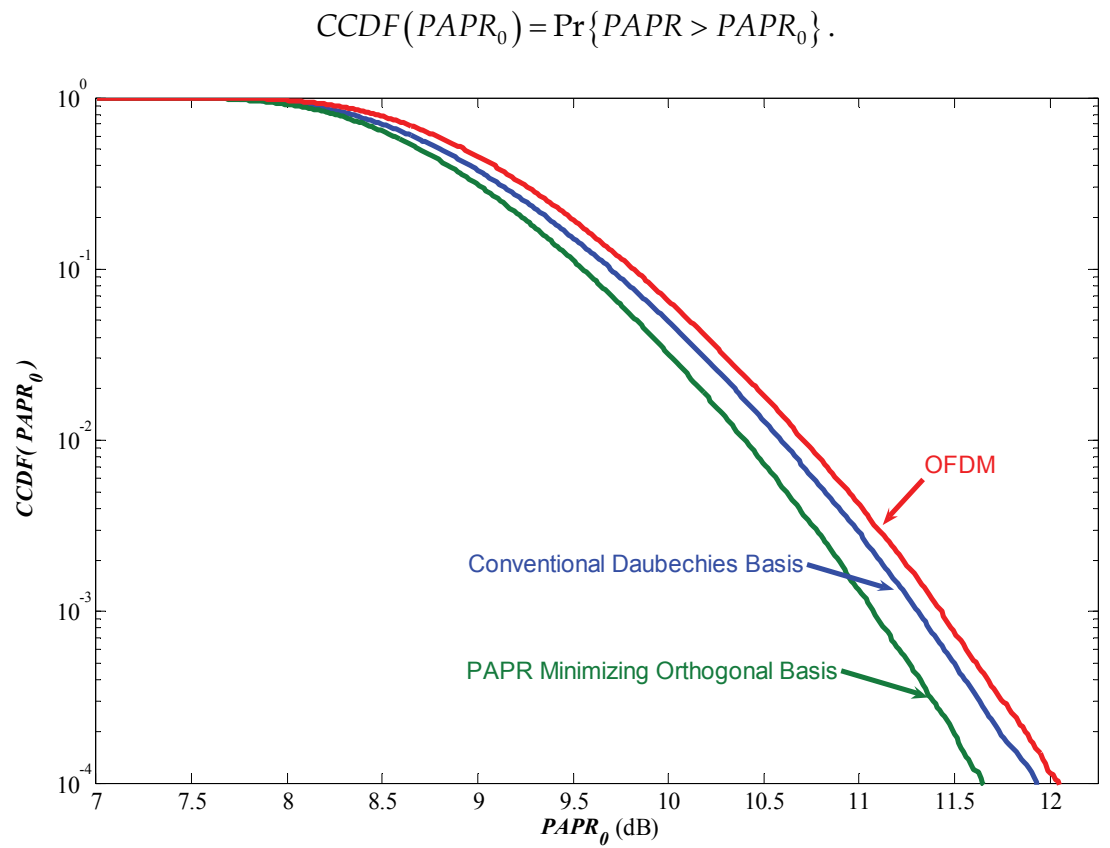

Fig. 3. CCDF performance comparison

The CCDF performance comparison between the three multi-carrier systems is presented in Figure 3. From Figure 3, we note that the PAPR minimizing orthogonal basis functions achieve PAPR reductions of $0.3 \mathrm{~dB}$ over the conventional Daubechies basis functions and 0.4 $\mathrm{dB}$ over OFDM. It should be emphasized that these performance gains are attained with no need for side information to be sent to the receiver, no distortion, and no loss in bandwidth efficiency. Furthermore, if additional PAPR reduction is desired, the PAPR minimizing orthogonal basis functions can also be combined with some of the PAPR reduction methods surveyed in (Han \& Lee, 2005) and (Jiang \& Wu, 2008).

Next, we compare the bit error rate (BER) performances of the three multi-carrier systems under consideration. In the BER comparisons, we utilize the Rapp's model to characterize the high power amplifier with the non-linear characteristic parameter chosen as 2 and the saturation amplitude set to 3.75 (van Nee \& Prasad, 2000). Furthermore, a 10-path channel with an exponentially decaying power delay profile and a root mean square delay spread of $50 \mathrm{~ns}$ is assumed. The BER results as a function of the normalized signal-to-noise ratio (SNR) are shown in Figure 4. From the figure, it is noted that at a target BER of $3 \times 10^{-4}$, the PAPR minimizing orthogonal basis functions achieve an SNR gain of $2.9 \mathrm{~dB}$ over the conventional Daubechies basis functions. The corresponding SNR gain over OFDM is $6.5 \mathrm{~dB}$.

We next quantify the out-of-band power emissions associated with the PAPR minimizing orthogonal basis functions, the conventional Daubechies basis functions, and the OFDM system. This is done by analyzing the adjacent channel power ratio (ACPR)-CCDF corresponding to the three different schemes. The ACPR-CCDF is defined as 


$$
C C D F\left(A C P R_{0}\right)=\operatorname{Pr}\left\{A C P R>A C P R_{0}\right\} .
$$

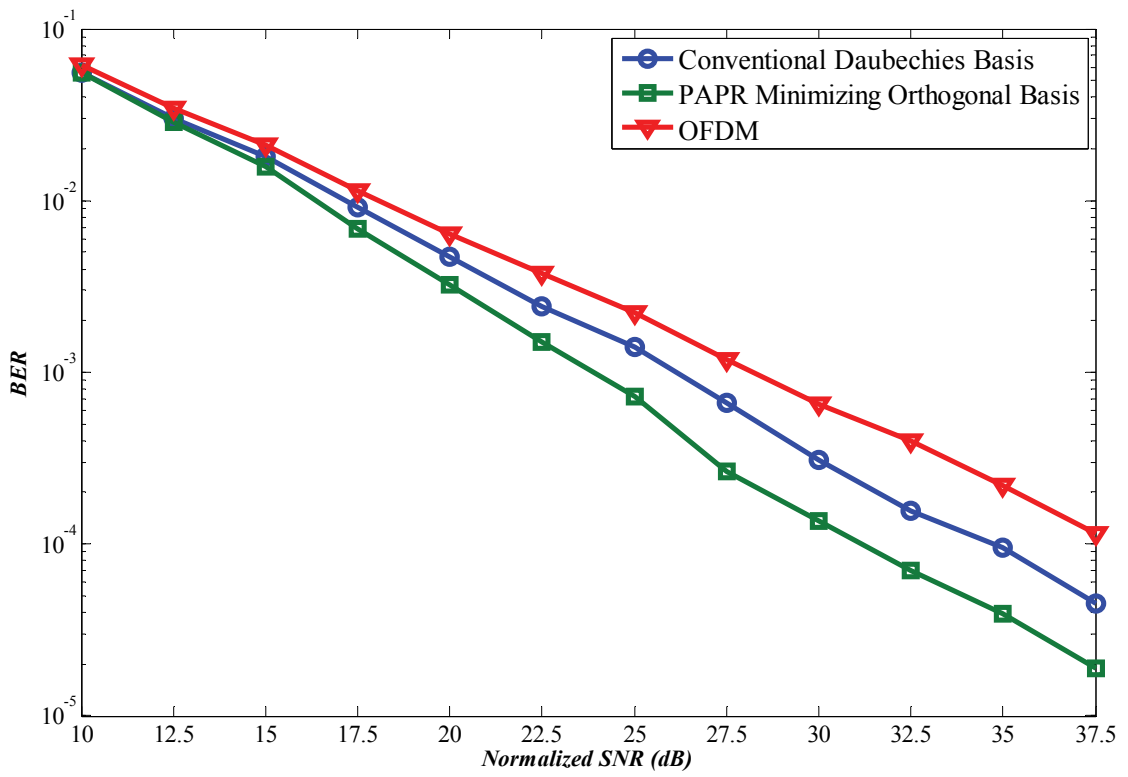

Fig. 4. BER performance comparison

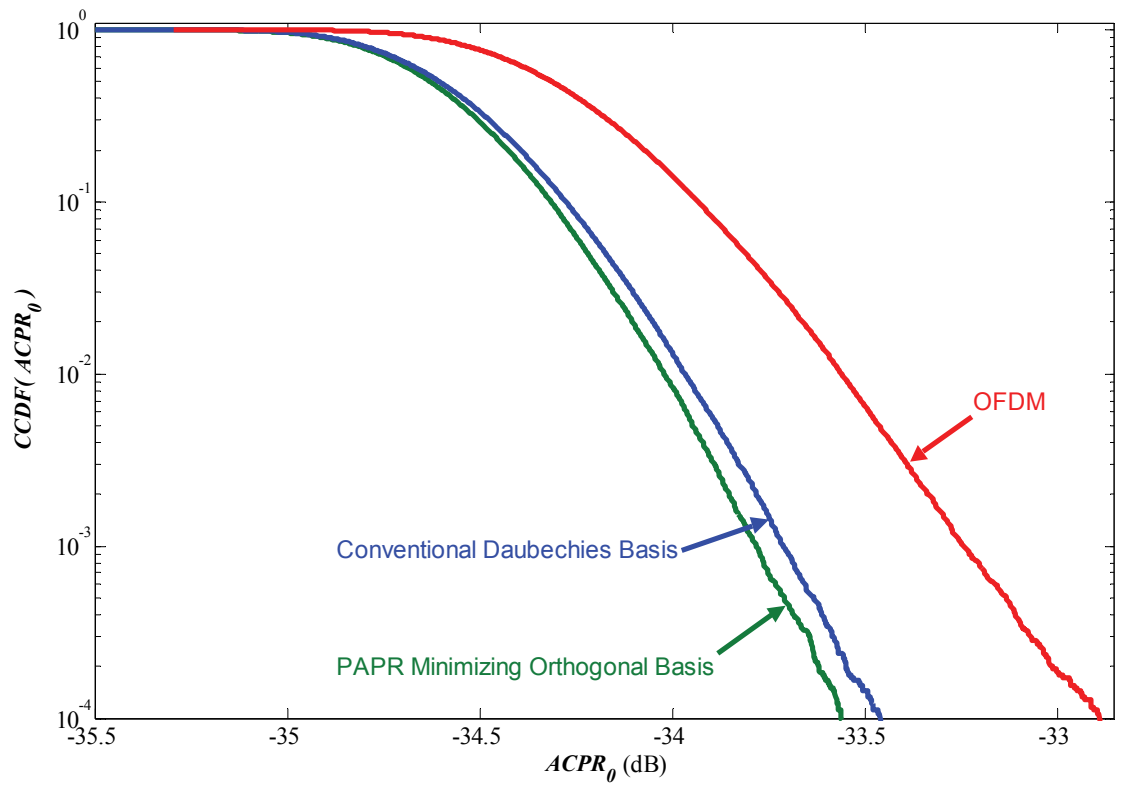

Fig. 5. ACPR-CCDF performance comparison 


\begin{tabular}{|c|c|c|c|c|c|}
\hline Method & $\begin{array}{l}\text { PAPR minimizing } \\
\text { orthogonal basis } \\
\text { functions }\end{array}$ & $\begin{array}{l}\text { PTS and } \\
\text { SLM }\end{array}$ & $\begin{array}{c}\text { Using different } \\
\text { Daubechies } \\
\text { pulse shapes }\end{array}$ & $\begin{array}{l}\text { Adaptive } \\
\text { threshold } \\
\text { companding }\end{array}$ & $\begin{array}{r}\text { Low } \\
\text { amplit } \\
\text { thresh }\end{array}$ \\
\hline $\begin{array}{l}\text { PAPR Reduction } \\
\text { Capability }\end{array}$ & Moderate & High & High & High & Mod \\
\hline $\begin{array}{l}\text { Implementation } \\
\text { Complexity }\end{array}$ & $\begin{array}{c}\text { Low (since the PAPR } \\
\text { minimizing } \\
\text { orthogonal basis } \\
\text { functions are } \\
\text { designed offline) }\end{array}$ & High & $\begin{array}{c}\text { High (due to an } \\
\text { increase in the } \\
\text { wavelet index) }\end{array}$ & Low & \\
\hline Spectral Regrowth & No & No & No & Yes & \\
\hline $\begin{array}{l}\text { BER Degradation } \\
\text { in AWGN }\end{array}$ & No & No & No & Yes & \\
\hline $\begin{array}{l}\text { Applicability in } \\
\text { Multipath Fading }\end{array}$ & Performs well & $\begin{array}{c}\text { Performs } \\
\text { well }\end{array}$ & \begin{tabular}{|c|} 
May not be \\
feasible due to \\
a high \\
complexity
\end{tabular} & $\begin{array}{l}\text { May not be } \\
\text { practical in a } \\
\text { multipath } \\
\text { environment }\end{array}$ & $\begin{array}{r}\text { Not pra } \\
\text { in a mul } \\
\text { environ }\end{array}$ \\
\hline Distortionless & Yes & Yes & Yes & Yes (for AWGN) & \\
\hline Side Information & Not required & Required & Not required & Required & Not 1 \\
\hline
\end{tabular}


Figure 5 shows the ACPR-CCDF results which are generated using the same power amplifier model used to generate the results of Figure 4. For a CCDF probability of $10^{-4}$, we note from Figure 5 that the PAPR minimizing orthogonal basis functions achieve an ACPR reduction of approximately $0.67 \mathrm{~dB}$ over the OFDM system. It should be noted that when compared to OFDM, the PAPR minimizing orthogonal basis functions reduce the ACPR by reducing the out-of-band power emissions introduced by the non-linear power amplifier. Furthermore, it is also noted from Figure 5 that the PAPR minimizing orthogonal basis functions yield a $0.1 \mathrm{~dB}$ ACPR reduction over the conventional Daubechies basis functions. This ACPR reduction is achieved mainly due to the superior PAPR reduction performance associated with the PAPR minimizing orthogonal basis functions when compared to the conventional Daubechies basis functions.

Lastly, in Table 1, we provide a qualitative comparison of the PAPR minimizing orthogonal basis functions presented in Section 4 with the other PAPR reduction techniques overviewed in Section 3. It should be noted that although the PAPR minimizing orthogonal basis functions yield a marginal PAPR reduction, this PAPR reduction is achieved without the disadvantages associated with the other techniques. Moreover, if a high PAPR reduction is desired, the PAPR reducing orthogonal basis functions also offer the flexibility to be combined with other PAPR reduction methods such as PTS, SLM, etc.

\section{Conclusions}

In this chapter, a PAPR reduction method for WPM multicarrier systems based on the orthogonal basis function design approach is presented. Firstly, we provide an overview of the WPM system and survey some PAPR reduction methods already proposed in the literature for WPM systems. Next, we derive a new PAPR upper bound that applies the triangular inequality to the WPM transmitter output signal. Using the new PAPR upper bound derived, design criteria for PAPR minimizing orthogonal basis functions are next formulated. The performance of the PAPR minimizing orthogonal basis functions is compared to those of the conventional Daubechies basis functions and OFDM. These comparisons show that the PAPR minimizing orthogonal basis functions outperform both the conventional Daubechies basis functions and OFDM. Furthermore, we also provide a qualitative comparison between the PAPR minimizing orthogonal basis functions and the existing PAPR reduction techniques. Through this comparison, it is shown that the PAPR minimizing orthogonal basis functions reduce the PAPR without the disadvantages associated with the other techniques.

\section{References}

Akho-Zhieh, M. M. \& Ugweje, O. C. (2008). Diversity performance of a wavelet-packetbased multicarrier multicode CDMA communication system. IEEE Transactions on Vehicular Technology, vol. 57, no. 2, pp. 787-797.

Baro, M. \& Ilow, J. (2007a). PAPR reduction in wavelet packet modulation using tree pruning. IEEE 65 th Vehicular Technology Conference (VTC-Spring), pp. 1756-1760.

Baro, M. \& Ilow, J. (2007b). Improved PAPR reduction for wavelet packet modulation using multi-pass tree pruning. IEEE $18^{\text {th }}$ International Symposium on Personal, Indoor and Mobile Radio Communications (PIMRC), pp. 1-5. 
Burrus, C. S., Gopinath, R. A., \& Guo, H. (1998). Introduction to Wavelets and Wavelet Transforms: A Primer, Prentice Hall.

Cimini, L. J. \& Sollenburger, N. R. (2000). Peak-to-average power ratio reduction of an OFDM signal using partial transmit sequences. IEEE Communications Letters, vol. 4, no. 3, pp. 86-88.

Daly, D., Heneghan, C., Fagan, A., \& Vetterli, M. (2002). Optimal wavelet packet modulation under finite complexity constraint. IEEE Int'l Conf. Acoustics, Speech, and Signal Processing (ICASSP), vol. 3, pp. 2789-2792.

Daubechies, I. (1992). Ten lectures on wavelets, SIAM.

Gautier, M., Lereau, C., Arndt, M., \& Lienard, J. (2008). PAPR analysis in wavelet packet modulation. IEEE International Symposium on Communications, Control, and Signal Processing (ISCCSP), pp. 799-803.

Han, S. H. \& Lee, J. H. (2005). An overview of peak-to-average power ratio reduction techniques for multicarrier transmission. IEEE Wireless Communications, vol. 12, no. 2, pp. 56-65.

Jiang, T. \& Wu, Y. (2008). An overview: peak-to-average power ratio reduction techniques for OFDM signals. IEEE Transactions on Broadcasting, vol. 54, no. 2, pp. 257-268.

Lakshmanan, M. K. \& Nikookar, H. (2006). A review of wavelets for digital communication. Wireless Personal Communications, vol. 37, no. 3-4, pp. 387-420.

Lakshmanan, M. K., Budiarjo, I., \& Nikookar, H. (2007). Maximally frequency selective wavelet packets based multi-carrier modulation scheme for cognitive radio systems. IEEE Global Telecommunications Conference, pp. 4185-4189.

Lakshmanan, M. K., Budiarjo, I., \& Nikookar, H. (2008). Wavelet packet multi-carrier modulation MIMO based cognitive radio systems with VBLAST receiver architecture. IEEE Wireless Communications and Networking Conference, pp. 705-710.

Le, N. T., Muruganathan, S. D., \& Sesay, A. B. (2008). Peak-to-average power ratio reduction for wavelet packet modulation schemes via basis function design. IEEE Vehicular Technology Conference (VTC-Fall), pp. 1-5.

Lindsey, A. R. (1995). Generalized orthogonal multiplexed communications via wavelet packet bases. Ph.D. Dissertation, Ohio University.

Rostamzadeh, M. \& Vakily, V. T. (2008). PAPR reduction in wavelet packet modulation. IEEE $5^{\text {th }}$ International Multi-Conference on Systems, Signals and Devices (SSD), pp. 1-6.

Rostamzadeh, M., Vakily, V. T., \& Moshfegh, M. (2008). PAPR reduction in WPDM and OFDM systems using an adaptive threshold companding scheme. IEEE $5^{\text {th }}$ International Multi-Conference on Systems, Signals and Devices (SSD), pp. 1-6.

Sandberg, S. D. \& Tzannes, M. A. (1995). Overlapped discrete multitone modulation for high speed copper wire communications. IEEE Journal of Selected Areas in Communications, vol. 13, no. 9, pp. 1571-1585.

van Nee, R. \& Prasad, R. (2000). OFDM for Wireless Multimedia Communications, Artech House.

Zhang, H., Yuan, D., \& Zhao, F. (2005). Research of PAPR reduction method in multicarrier modulation system. IEEE International Conference on Communications, Circuits and Systems (ICCCAS), vol. 1, pp. 91-94. 


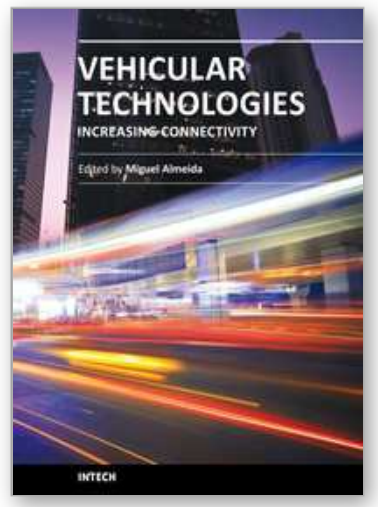

\author{
Vehicular Technologies: Increasing Connectivity \\ Edited by Dr Miguel Almeida
}

ISBN 978-953-307-223-4

Hard cover, 448 pages

Publisher InTech

Published online 11, April, 2011

Published in print edition April, 2011

This book provides an insight on both the challenges and the technological solutions of several approaches, which allow connecting vehicles between each other and with the network. It underlines the trends on networking capabilities and their issues, further focusing on the MAC and Physical layer challenges. Ranging from the advances on radio access technologies to intelligent mechanisms deployed to enhance cooperative communications, cognitive radio and multiple antenna systems have been given particular highlight.

\title{
How to reference
}

In order to correctly reference this scholarly work, feel free to copy and paste the following:

Ngon Thanh Le, Siva D. Muruganathan and Abu B. Sesay (2011). Peak-to-Average Power Ratio Reduction for Wavelet Packet Modulation Schemes via Basis Function Design, Vehicular Technologies: Increasing Connectivity, Dr Miguel Almeida (Ed.), ISBN: 978-953-307-223-4, InTech, Available from:

http://www.intechopen.com/books/vehicular-technologies-increasing-connectivity/peak-to-average-power-ratioreduction-for-wavelet-packet-modulation-schemes-via-basis-function-desig

\section{INTECH}

open science | open minds

\section{InTech Europe}

University Campus STeP Ri

Slavka Krautzeka 83/A

51000 Rijeka, Croatia

Phone: +385 (51) 770447

Fax: +385 (51) 686166

www.intechopen.com

\section{InTech China}

Unit 405, Office Block, Hotel Equatorial Shanghai

No.65, Yan An Road (West), Shanghai, 200040, China

中国上海市延安西路 65 号上海国际贵都大饭店办公楼 405 单元

Phone: +86-21-62489820

Fax: +86-21-62489821 
(C) 2011 The Author(s). Licensee IntechOpen. This chapter is distributed under the terms of the Creative Commons Attribution-NonCommercialShareAlike-3.0 License, which permits use, distribution and reproduction for non-commercial purposes, provided the original is properly cited and derivative works building on this content are distributed under the same license. 